
\title{
25 Research Square \\ Comparison of Perforated Appendicitis Prior to and Post quarantine during COVID19 Pandemic
}

\author{
Haley Lehman ( $\nabla$ hlehman1@hfhs.org ) \\ Henry Ford Macomb https://orcid.org/0000-0001-8204-1196 \\ Jeffrey Turnbull \\ Michigan State University College of Osteopathic Medicine \\ Janice Prescod \\ Michigan State University College of Osteopathic Medicine \\ Trevor Gohl \\ Michigan State University College of Osteopathic Medicine \\ Peter Lopez \\ Henry Ford Macomb Hospital \\ Robert Jarski
}

\section{Research Article}

Keywords: COVID-19, perforated appendicitis

Posted Date: September 15th, 2021

DOl: https://doi.org/10.21203/rs.3.rs-902442/v1

License: (c) (1) This work is licensed under a Creative Commons Attribution 4.0 International License.

Read Full License 


\section{Abstract}

Background: The SARS-CoV-2 virus has caused patient aversion to health care facilities for fear of contracting the virus. It has been shown that there has been a decreased amount of emergency department visits since the pandemic started ${ }^{1}$, with an increased presentation of conditions with progressed pathology. At our community hospital, it was anecdotally noted that there was an increase in the number of patients presenting with perforated appendicitis during this time. Our hypothesis is that more patients presented with the progressed finding of perforated appendicitis during rather than prior to the pandemic because of fear of exposure to COVID-19 in the health care setting.

Methods: Retrospective chart review of cases of perforated appendicitis at a local community hospital during March 10-December 31, 2019 (cohort A) and March 10-December 31, 2020 (cohort B).

Results: A total of three hundred and fifty-two eligible patients received treatment for acute appendicitis at our community hospital. In Cohort A (prior to the pandemic), there were 53 perforations out of 188 patients $(53 / 188)-28.2 \%$. In cohort B (during the pandemic), there was 61 perforations out of 164 patients $(61 / 164)-37.2 \%$. Statistically, there was no difference in the rate of perforation between Cohort $A$ and Cohort $B$, approaching significance ( $p$-value $=0.072$ ). The median age of patients in Cohort $A$ was 38 and in Cohort B it was 43.5 years of age. There was no statistically significant differences between cohorts regarding age $(p<0.23)$ and sex $(p<0.8)$.

Conclusion: This study found that the number of patients who presented with perforated appendicitis during the pandemic did increase compared to pre-pandemic (37.2\% vs $28.2 \%)$ but this did not meet statistical significance $(p=0.072)$.

\section{Introduction}

SARS-CoV-2 (COVID-19) has had a major impact on the health care system worldwide. Of note, one of the most prominent effects has been avoidance of timely medical treatment secondary to fear of contracting the novel COVID-19 virus. Emergency departments internationally have reported significant decreases in volume in comparison to previous years [1, 2], attributing the lower census to this phenomenon. The impact of delayed presentation of certain conditions during the pandemic, especially those requiring surgical intervention, has yet to be truly elucidated.

At our institution, it was anecdotally noted that many patients who presented to the hospital during the COVID-19 pandemic had perforated appendicitis. Perforated appendicitis is associated with increased morbidity for patients, with delays in presentation increasing mortality up to $4 \%[3,4]$. This raises the concern of an increased population of patients with progressed disease, adding a significant burden to the health care system.

If patients with appendicitis in fact delayed presentation to health care settings secondary to fear, it would theoretically increase the incidence of perforated appendicitis. This study aims to compare the 
rates of perforated appendicitis during the COVID-19 pandemic to the previous year to evaluate if the fear of seeking medical treatment has increased the number of perforated appendicitis cases seen during the pandemic.

\section{Methods}

This is a single center retrospective chart review that evaluated the number of perforated appendicitis cases during the first nine months of the pandemic and the nine months prior. IRB approval was obtained at Henry Ford Macomb Hospital in Clinton Twp Michigan. The first COVID-19 case in Michigan was diagnosed on March 10, 2020 and a stay-at-home order was established on March 24, 2020 [5]. Every patient with the diagnosis of acute appendicitis between the dates of March 10-December 31, 2019 (cohort A) and March 10-December 31, 2020 (cohort B) was evaluated. A total of 377 patients were evaluated for the diagnosis of acute appendicitis and 22 patients were excluded from evaluation during these time frames.

\section{Exclusion Criteria}

Patients were excluded from the study if appendicitis was ruled out based on physical exam, if the patient was transferred to another facility, if they had a normal appendix on diagnostic laparoscopy, or if there was concern/diagnosis of neoplasm. In our patient population, five patients were transferred to another facility, eight patients had normal appendix on diagnostic laparoscopy, and acute appendicitis was ruled out on physical exam in seven patients. One patient had an incidental appendectomy as a part of another procedure, and three patients had concern for appendiceal carcinoma. A total of 352 patients were included in this study. Cohort A included 188 patients and cohort B included 164 patients.

\section{Data Collection}

Age, sex, race, comorbidities including hypertension, hyperlipidemia, diabetes mellitus, coronary artery disease, renal failure, and obesity, COVID status, imaging findings, operative findings, length of stay, and complications were studied. Perforated appendicitis was defined as findings on CT scan if applicable and/or intraoperatively. Post-operative abscess was observed on CT scan or in an additional operation. lleus was defined as delayed bowel function requiring length of stay longer than two days or requiring readmission. Surgical site infection was defined as erythema or drainage from an incision requiring treatment with antibiotics. Bleeding was diagnosed intraoperatively after initial intervention.

\section{Statistical Analysis}

Categorical data were summarized as counts and percentages, and numerical data (patient age) as means with corresponding standard deviations. Between-group mean differences were compared by calculating t-tests for independent variables. Categorical data were compared using the chi-square test for association or Fisher's exact test, as appropriate. Throughout this study, a p-value $<0.05$ (two-tail) was considered statistically significant. Following initial data entry using Microsoft Excel, Minitab 19 
Statistical Software (2019), State College, PA: Minitab, Inc. (www.minitab.com) was used for performing the analyses.

\section{Results}

In this study, a total of three hundred and fifty-two eligible patients received a diagnosis and treatment for acute appendicitis. In Cohort A, there were 53 perforations out of 188 patients before Covid-19 (53/188) $28.2 \%$. In cohort B, there was 61 perforations out of 164 patients during Covid-19 (61/164) $-37.2 \%$. Although trending toward significance, there was no statistical difference in the rate of perforation between Cohort A and Cohort $B(p$-value $=0.072)$. There was no difference in comorbidities between Cohort A and B. (see table 1)

The median age of patients in Cohort A was 38 and in Cohort B it was 43.5 years of age. There was no statistically significant differences between cohorts regarding age $(p<0.23)$ and sex $(p<0.8)$. See table 1 . Between groups, 182 (51.70\%) were male and 170 (48.29\%) were female. In cohort A, patients' age ranged from nine years to ninety years old, with a median age of thirty-eight years. In cohort B, patient age ranged from thirteen years to eighty-nine years old, with a median age of 43.5 years. 166 (93.78\%) patients identified as white in cohort A and 134 (84.27\%) patients identified as white in cohort B. There was a statistically significant difference in the number of non-white patients in Cohort $B(15.7 \%)$ than Cohort A (6.2\%) [p = 0.017].

Patients were screened for existing comorbidities such as obesity, hypertension, hyperlipidemia, diabetes mellitus, coronary artery disease, and renal failure. Our data demonstrated no significant differences related to these comorbidities between cohorts (Table 1). Routine COVID-19 testing was only employed to patients managed during the pandemic in cohort B. Four patients out of 164 patients $(1.22 \%)$ tested positive for COVID-19.

Diagnosis of acute appendicitis was based on clinical symptomatology, CT findings, and intraoperative observation and surgical pathology. Decisions related to type of treatment were based on CT findings and attending physician discretion. $90.57 \%$ of patients between both cohorts underwent laparoscopic appendectomy. Other treatments included open appendectomy, percutaneous drainage, medical management with antibiotics, and laparoscopic drainage. Patients requiring conversion from laparoscopic approach to open approach were categorized as open appendectomy. No statistically significant differences existed between cohorts related to type of management $(p<0.685)$.

Length of stay was reported as the number of days between discharge and admission. The average length of stay for cohort A and cohort B was 2.06 and 1.91 days respectively. Comparing this data between groups using chi-square analysis demonstrated statistical significance $(p=0.006)$, with patients in cohort B staying fewer days on average (Fig. 2). Post-operative complications were evaluated including surgical site infection, ileus, and bleeding. In total, nineteen (5.39\%) patients experienced ileus, two $(0.57 \%)$ surgical site infection, and three $(0.85 \%)$ experienced postoperative bleeding. No significant 
differences in post-operative complications existed between groups. Cohort A had an overall complication rate of $16.8 \%$ and Cohort B had an overall complication rate of $17.1 \%$ (Fig. 3).

\section{Discussion}

This study evaluated the number of cases of perforated appendicitis in a small community hospital during the pandemic in 2020 (Cohort A) compared to the year prior (Cohort B). Cohort A had an increased number of patients who presented with a diagnosis of appendicitis in comparison to cohort B. It has been well documented that emergency department visits declined during the pandemic [1], which could possibly account for the larger sample size in cohort A. It is also possible that patients with uncomplicated appendicitis with mild symptoms did not present to health care facilities as symptoms were not severe enough to seek care during high-risk times. Demographics were similar in both cohorts, except for the larger number of African Americans who presented during pandemic times $(p<0.01)$. Of note, only $2.4 \%$ of patients in cohort B were infected with COVID-19 at time of presentation. Although minimal data has been reported on this topic, there does not seem to be an association with coronavirus and appendicitis. $28 \%$ of patients in cohort A had perforated appendicitis upon presentation, in comparison with $37 \%$ of patients in cohort B. Although trending towards significance, there was not a statistically significant difference in perforation between cohorts $(p<0.072)$. Unexpectedly, cohort $A$ had a longer average length of stay than cohort $B(p<0.006)$. Although only speculation, this could be due to the push to limit hospital stays in the pandemic, resulting in expedited discharge of patients in cohort $B$. Individual complications did not significantly differ in either cohort, showing no correlation with the pandemic times and increasing morbidity of the presenting pathology.

It has become a concern that patients may not be seeking the medical care in a timely manner secondary to the increased anxiety surrounding the virus [1]. This notion has prompted investigation into the possibility of delayed presentation in serious illness including ischemic stroke and myocardial infarction, showing increases delay of presentation during the pandemic $[6,7]$. The idea of possibly delaying care secondary to patient fear has sparked investigation into surgical emergencies as well. Patel et al has speculated that there may be an association between COVID-19 infection and perforated diverticulitis [8], while Karam et al describes a case of perforated rectal cancer as a result of delayed presentation during this time period [9]. Of the studies that have been published related to appendicitis during the pandemic, there has not been an increased incidence of perforated appendicitis reported. Turanli et al showed in their patient population that there was no clear increase in the number of patients with perforated appendicitis in a similar time period [9]. Another study in Germany that showed a decrease in the presentation of uncomplicated appendicitis also showed no difference in incidence of perforated appendicitis [10], findings that are similar to the results in our study. Although pandemic times have not appeared to increase the incidence of perforated appendicitis to the point of statistical significance, our study shows a trend toward significance during this time period, which demonstrates the need for further investigation on this topic. 
There are limitations to our study. Due to the retroactive nature of data collection, we were unable to reliably specify duration of symptoms, making it difficult to extrapolate delayed presentation.

Additionally, because data was collected from one community hospital, our patient population may have been limited by social and cultural pressure specific to the demographic studied. Moreso, the time constraints for analysis of nine months may have limited our sample size. As our results trended toward significance $(p<0.07)$, it is possible that a larger patient sample size would have allowed for the data to reach statistical significance. Other factors that may have increased variance in our study include the wide age range of patients within our analysis. Other studies have shown an increased incidence of perforated appendicitis in the pediatric population during the COVID-19 pandemic [11], a demographic of patients which is not as commonly treated at this community center. Further research in the pediatric population in this area could demonstrate different findings.

\section{Conclusion}

This study found that the number of patients who presented with perforated appendicitis during the pandemic did increase compared to pre-pandemic (37.2\% vs $28.2 \%)$, but this did not meet statistical significance $(p=0.072)$.

\section{Declarations}

Competing interests: The authors declare no competing interests.

\section{References}

1. Mantica, G., Riccardi, N., Terrone, C., \& Gratarola, A. (2020). Non-COVID-19 visits to emergency departments during the pandemic: the impact of fear. In Public Health. https://doi.org/10.1016/j.puhe.2020.04.046

2. Köhler, F., Acar, L., van den Berg, A., Flemming, S., Kastner, C., Müller, S., Diers, J., Germer, C. T., Lock, J. F., L'hoest, H., Marschall, U., \& Wiegering, A. (2021). Impact of the COVID-19 pandemic on appendicitis treatment in Germany-a population-based analysis. Langenbeck's Archives of Surgery. https://doi.org/10.1007/s00423-021-02081-4

3. Bhangu, A., Søreide, K., Di Saverio, S., Assarsson, J. H., \& Drake, F. T. (2015). Acute appendicitis: Modern understanding of pathogenesis, diagnosis, and management. In The Lancet. https://doi.org/10.1016/S0140-6736(15)00275-5

4. Margenthaler, J. A., Longo, W. E., Virgo, K. S., Johnson, F. E., Oprian, C. A., Henderson, W. G., Daley, J., \& Khuri, S. F. (2003). Risk Factors for Adverse Outcomes After the Surgical Treatment of Appendicitis in Adults. Annals of Surgery. https://doi.org/10.1097/01.sla.0000074961.50020.f8

5. Haddad, Ken. "Michigan Coronavirus Timeline: Key Dates, COVID-19 Case Tracking, State Orders." Click on Detroit, 9 Apr. 2020, . 
6. Schirmer, C. M., Ringer, A. J., Arthur, A. S., Binning, M. J., Fox, W. C., James, R. F., Levitt, M. R., Tawk, R. G., Veznedaroglu, E., Walker, M., \& Spiotta, A. M. (2020). Delayed presentation of acute ischemic strokes during the COVID-19 crisis. Journal of Neurolnterventional Surgery. https://doi.org/10.1136/neurintsurg-2020-016299

7. Braiteh, N., Rehman, W. ur, Alom, M., Skovira, V., Breiteh, N., Rehman, I., Yarkoni, A., Kahsou, H., \& Rehman, A. (2020). Decrease in acute coronary syndrome presentations during the COVID-19 pandemic in upstate New York. In American Heart Journal. https://doi.org/10.1016/j.ahj.2020.05.009

8. Patel, Pratik MD¹; Phan, Ethan MD¹; Pona, Adrian MD¹; Mao, Yuxuan MD, MSc ${ }^{2}$ S1625 Acute Perforated Diverticulitis as a Potential Complication of SARS-CoV-2 (COVID-19), The American Journal of Gastroenterology: October 2020 - Volume 115 - Issue - p S834 doi:

10.14309/01.ajg.0000708548.90106.60

9. https://onlinelibrary.wiley.com/doi/10.1111/ans.16104

10. Turanli, S., \& Kiziltan, G. (2021). Did the COVID-19 Pandemic Cause a Delay in the Diagnosis of Acute Appendicitis? World Journal of Surgery. https://doi.org/10.1007/s00268-020-05825-3

11. Fisher JC, Tomita SS, Ginsburg HB, Gordon A, Walker D, Kuenzler KA. Increase in Pediatric Perforated Appendicitis in the New York City Metropolitan Region at the Epicenter of the COVID-19 Outbreak. Ann Surg. 2021;273(3):410-415. doi:10.1097/SLA.0000000000004426

\section{Table}

Due to technical limitations, the table can only be accessed as a download in the supplementary files section.

\section{Figures}




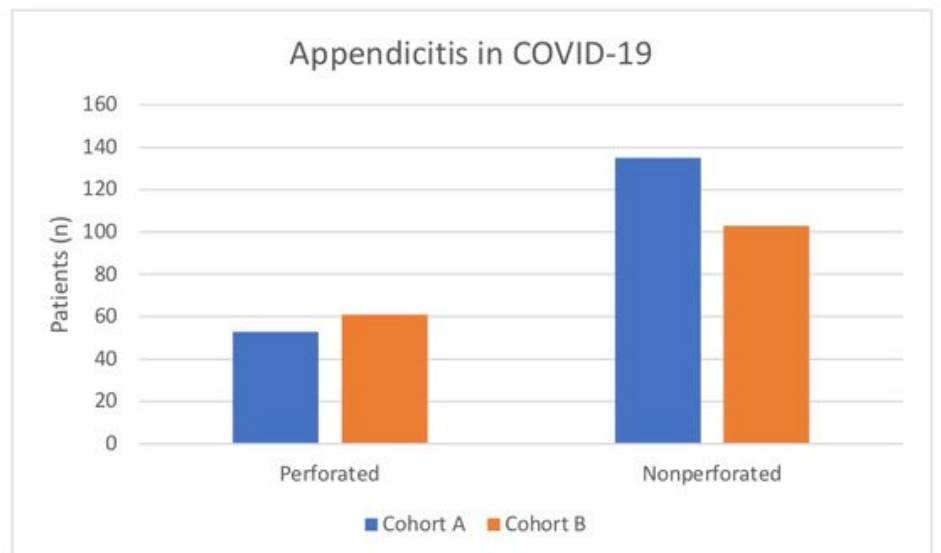

Figure 1.

\section{Figure 1}

Figure 1. Patients with perforated appendicitis vs nonperforated appendicitis in Cohorts A and B. Comparison between perforation in each cohort showed no statistically significant difference $(p<0.07)$. 


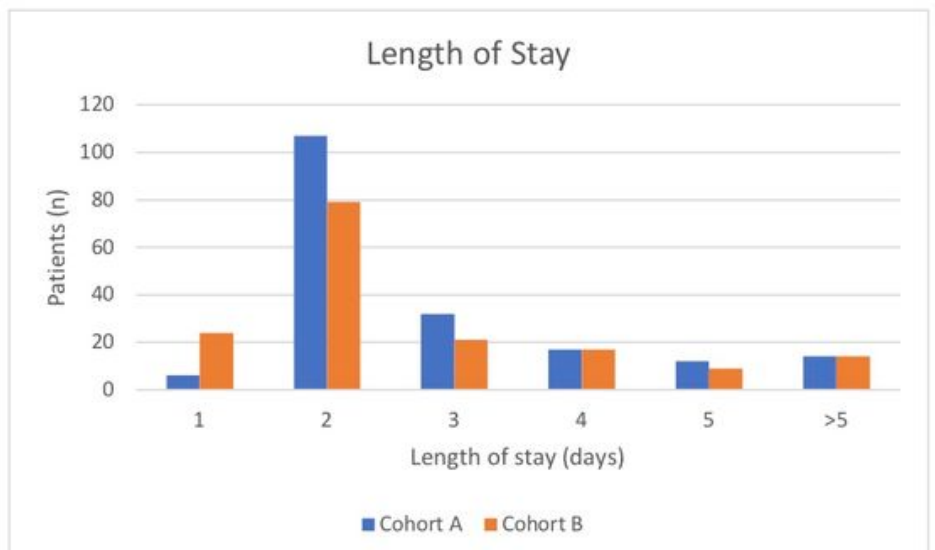

Figure 2 .

\section{Figure 2}

Figure 2. Length of stay in days defined as groups 1 day-greater than 5 days. Length of stay longer in cohort A vs. cohort $B(p<0.006)$ 


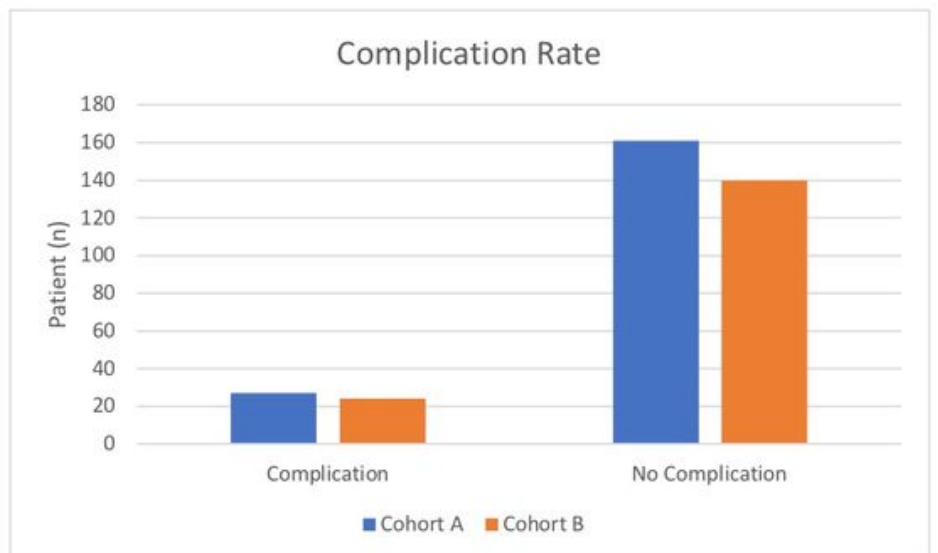

Figure 3.

Figure 3

Figure 3. Overall complication rates compared between both cohorts. Cohort A had an overall complication rate of $16.8 \%$ and Cohort $\mathrm{B}$ had an overall complication rate of $17.1 \%$.

\section{Supplementary Files}


This is a list of supplementary files associated with this preprint. Click to download.

- Table1.jpg 